\title{
Matrix stiffness regulates the proliferation, stemness and chemoresistance of laryngeal squamous cancer cells
}

\author{
LIAN HUI, JINGRU ZHANG, XIAOXU DING, XING GUO and XUEJUN JIANG
}

Department of Otolaryngology, The First Hospital of China Medical University, Shenyang, Liaoning 110001, P.R. China

Received December 9, 2016; Accepted February 2, 2017

DOI: $10.3892 /$ ijo.2017.3877

\begin{abstract}
Increasing evidence shows that matrix stiffness plays a critical role in affecting the phenotype and behavior of tumor cells. We report that matrix stiffness significantly regulated the proliferation and chemotherapeutic response of Hep-2 cells. Increasing substrate stiffness promotes the proliferation of Hep- 2 cells. When cultured on soft gels, Hep-2 cells expressed higher level of stem cell markers. Following treatment with cisplatin or 5-FU, we observed reduced apoptosis in Hep-2 cells cultured on soft supports. Sox 2 is essential for the chemoresistance of Hep-2 cells cultured in soft stiffness. Our results demonstrated that Sox 2 promotes chemoresistance of Hep- 2 cells in soft stiffness via upregulating the expression of ABCG2. Our findings will provide a new platform for the future design of anticancer drugs based on the biophysical properties of the tumor site.
\end{abstract}

\section{Introduction}

Laryngeal squamous cell carcinoma (LSCC) is one of the leading malignant cancers of the head and neck and accounts for $\sim 2.4 \%$ of newly diagnosed malignancies worldwide every year $(1,2)$. Despite the development of conventional approaches of surgery, chemotherapy and radiotherapy, the 5-year survival rate of patients with advanced tumor remains poor, due to recurrence, metastasis and multiple drug resistance (MDR) (3). To identify more potential drugs and improve the survival and life quality of LSCC patients, clarifying the mechanisms of LSCC growth, metastasis and drug resistance remain an urgent need.

It is known that many solid tumors is significantly stiffer than normal tissues because of the stiffer stroma formed from extracellular matrix crosslinking and collagen deposition (4-6). Recently studies reported that tumor growth and metastasis are affected by both biochemical and physical properties

Correspondence to: Dr Lian Hui, Department of Otolaryngology, The First Hospital of China Medical University, 155 Nanjing Bei Street, Shenyang, Liaoning 110001, P.R. China

E-mail: huilian613@outlook.com

Key words: matrix stiffness, laryngeal squamous cancer cells, chemoresistance, Sox 2, ABCG2 of the tumor microenvironment (4-8). There is increasing evidence that the tumor mechanical force is a critical factor of tumor initial, growth and metastasis. Schrader et al showed that matrix stiffness regulates the proliferation, dormancy and chemotherapeutic response of hepatocellular carcinoma cells (9). Epithelial-mesenchymal transition (EMT) is a critical program for tumor cell dissemination and metastasis $(10,11)$. High matrix stiffness could promote the nuclear translocation of TWIST1, inducing EMT in breast cancer and promoting tumor invasion and metastasis (12). In addition, soft matrix stiffness is known to maintain self-renew of stem cell, such as embryonic stem cell (ESC) and cancer stem cell (CSC) $(13,14)$. Previous studies have demonstrated that upregulation of stem cell-associated genes such as Sox2, Oct3/4, CD133 and c-kit were detected in B16 melanoma tumor cells cultured in soft substrate (14). Moreover, Tajik et al showed that mechanical force induced the transcription upregulation through forceinduced direct stretching of chromatin, leading to a better understanding of how directional forces influence the growth of cancer cells (15). However, the role of mechanical factors in modulating the growth and progression of LSCC remain poorly defined. In the present study, we employed a collagencoated polyacrylamide hydrogel system to understand how matrix stiffness regulates the growth of LSCC. Although various cancer therapy strategies have been greatly developed and improved, drug resistance is still a challenging issue for patients. Here we further explore the effect of matrix stiffness on the chemosensitivity of LSCC.

\section{Materials and methods}

Cell culture. Human laryngeal squamous cell carcinoma Hep-2 cells were obtained from China Center for Type Culture Collection (CCTCC, Wuhan, China) and cultured according to the guidelines. Briefly, Hep-2 cells were cultured in RPMI-1640 medium with $10 \%$ fetal bovine serum (FBS), $100 \mathrm{U}$ of penicillin and $100 \mathrm{mg} / \mathrm{ml}$ streptomycin.

Antibodies and reagents. Anti-human CD29 PE and antihuman Ki67 APC were purchased from eBiosciences (La Jolla, CA, USA). Cyclin D1, cyclin D3, p-FAK, FAK, p-Akt, Akt, p-STAT3, STAT3, p-Erk, Erk, Sox 2, ABCG2 and $\beta$-actin antibodies were purchased from CST (Boston, MA, USA). Annexin V Apoptosis Detection kit was from BD Pharmingen (San Diego, CA, USA). 
Polyacrylamide gel preparation. Polyacrylamide (PA) gels of variable stiffness were prepared as previously described. Briefly, 25-mm glass coverslips were soaked using $0.1 \mathrm{~N}$ $\mathrm{NaOH}$ and air dried, functionalized using 3-aminopropyltriethoxysilane (Sigma-Aldrich), washed in distilled water and then soaked in $0.5 \%$ gluteraldehyde in PBS. Acrylamide/ bis-acrylamide gels polymerized between the functionalized coverslip and a glass slide treated with a hydrophobic silicon polymer (Rain-X ${ }^{\mathrm{TM}}$, SOPUS Products, Houston, TX, USA). The gels were then washed twice with $50 \mathrm{mM}$ HEPES and incubated with $1 \mathrm{mM}$ Sulfo-SANPAH (Thermo Scientific Pierce) in HEPES buffer under UV light for $10 \mathrm{~min}$. Excess Sulfo-SANPAH was removed by extensive washing in $50 \mathrm{mM}$ HEPES. A thin layer of collagen-I was then crosslinked to the gels at $37^{\circ} \mathrm{C}$ overnight, rinsed twice in $50 \mathrm{mM}$ HEPES buffer, and sterilized. Gels were soaked in serum-free culture media overnight before plating of cells.

Apoptosis assays. Hep-2 cells were cultured in 1 or $8 \mathrm{kPa}$ PA gels and treated with cisplatin $(5-20 \mu \mathrm{M})$ or 5 -FU $(5-20 \mu \mathrm{M})$ for $\leq 36 \mathrm{~h}$. In some cases, Hep-2 cells were transfected with Sox 2 or ABCG2 siRNA, and then cultured in $1 \mathrm{kPa}$ gels and treated with cisplatin or 5-fluorouracil (5-FU). Apoptosis was detected using the Annexin V/propidium iodide kit (BD Pharmingen) by flow cytometry.

CCK8 assay. CCK8 cell viability assay were performed according to the manufacturer's instructions. Briefly, Hep-2 cells were plated onto 1 or $8 \mathrm{kPa}$ gels and cultured with $1.8 \mathrm{ml}$ RPMI-1640 medium. Twelve hours later, cells were treated with a different concentration of cisplatin or 5-FU. After $24 \mathrm{~h}$ of treatment, $200 \mu \mathrm{l}$ WST-8 solution was added to each well, and the plates were incubated for an additional $1.5 \mathrm{~h}$ at $37^{\circ} \mathrm{C}$. Aliquot $(200 \mu \mathrm{l})$ of the solution from each well was transferred into a 96-well culture plate. The absorbance of each plate at $450 \mathrm{~nm}$ represented a direct correlation with the cell number in this analysis, and was measured by a standard microplate reader (Bio-Tek Instruments, Inc., Winooski, VT, USA).

Western blot analysis. Western blot analyses were performed following standard procedures. Hep-2 cell lysate proteins were extracted and analyzed by western blotting using antihuman-cyclin D1, anti-human-cyclin D3, anti-human-p-FAK, anti-human-FAK, anti-human-p-Akt, anti-human-Akt, antihuman-p-STAT3, anti-human-STAT3, anti-human-p-Erk, anti-human-Erk, anti-human-Sox 2, anti-human-ABCG2 and anti-human- $\beta$-actin antibodies, and then incubated with HRP-conjugated secondary antibodies. Proteins were visualized by ECL western blotting detection reagent (ECL kit; Pierce, Rockford, IL, USA).

Quantitative real-time PCR. Total RNA extracted from Hep-2 cells with TRIzol reagent (Invitrogen) and reverse transcribed using a High-Capacity RNA-to-cDNA kit (Applied Biosystems) according to standard procedures. RT-PCR was performed in a Bio-Rad real-time PCR detection system using SYBR Green Supermix (Bio-Rad, Carlsbad, CA, USA) and relative quantification was performed using the $\Delta \Delta \mathrm{Ct}$ method with $\beta$-actin as a reference. The primer sequences are shown in Table I.
Gene silencing experiments. siRNAs targeting human CD29, FAK or negative control siRNAs was transfected into Hep-2 cells using Lipofectamine RNAiMAX (Invitrogen) according to the manufacturer's instructions. Twenty-four hours after transfection, Hep- 2 cells were seeded on $8 \mathrm{kPa}$ gels for additional 48-h culture and the expressions of Ki67 were analyzed. In some cases, Sox 2, ABCG2 or negative control siRNAs was transfected into Hep-2 cells. Twenty-four hours after transfection, Hep-2 cells were seeded on $1 \mathrm{kPa}$ gels and treated with $10 \mu \mathrm{M}$ cisplatin or $10 \mu \mathrm{M} 5$-FU. Apoptosis of Hep-2 cells were analyzed by flow cytometry and CCK8 assay. All siRNAs were siGenome pools (Dharmacon). Knockdown of the indicated genes was verified by real-time PCR and western blot analysis.

Colony formation assay. Hep-2 cells were cultured for $48 \mathrm{~h}$ on either 1 or $8 \mathrm{kPa}$ gels. Cells were then left untreated or treated with cisplatin $(10 \mu \mathrm{M})$ or $5-\mathrm{FU}(10 \mu \mathrm{M})$ for $24 \mathrm{~h}$. Then the medium was changed and cultured for a further $48 \mathrm{~h}$. Cells were trypsinized and equal numbers re-plated at clonal density $(10,000$ cells/well) in 12-well plates in normal culture medium. After 10-day culture, cells were fixed in $4 \%$ paraformaldehyde and stained with $0.5 \%$ crystal violet.

Flow cytometry assay. For surface staining, Hep-2 cells were kept at $4^{\circ} \mathrm{C}$ and stained with PE-conjugated anti-29 (eBioscience). For intracellular staining, Hep-2 cells were first treated with Fix/ Perm solution and then stained with APC-conjugated anti-Ki67 (eBiosciences). Data were acquired on an BD FACSCanto II and analyzed with FlowJo software.

Statistical analysis. Data are presented as mean \pm SEM and assessed using Student's t-test analysis. P-values $<0.05$ were considered statistically significant. The analysis was conducted using the Graphpad 6.0 software. All experiments were repeated at least three times.

\section{Results}

Matrix stiffness modulates Hep-2 laryngeal cancer cell morphology and proliferation. To investigate the influence of matrix stiffness on the morphology and proliferation of Hep-2 laryngeal cancer cells, we seeded cells on collagen coated polyacrylamide (PA) gel with soft stiffness $(1 \mathrm{kPa})$ and stiff stiffness $(8 \mathrm{kPa})$. We found that Hep-2 displayed drastically different morphologies in gels with varying stiffness. After 24-h culture, cells maintained a rounded shape on soft gels of $1 \mathrm{kPa}$ and the cells cultured on $8 \mathrm{kPa}$ increased cell spreading along with increasing matrix stiffness (Fig. 1A). Consistently, Hep-2 showed increased cell spreading area (Fig. 1B) along with increased matrix stiffness. To test the effect of matrix stiffness on Hep-2 proliferation, we counted the cell numbers directly, and found an increase in total cell number with increasing matrix stiffness (Fig. 1C). We next found that the frequency of Ki67-stained Hep-2 cells increased markedly with increased matrix stiffness (Fig. 1D). Matrix stiffness had a corresponding effect on the expression of cell cycle regulators of G1 progression. We observed a strong reduction in the expression of cyclin D1 and cyclin D3 in cells cultured on soft gel (Fig. 1E). These results suggest that morphology and proliferation of Hep-2 were modulated by matrix stiffness. 
Table I. Sequences of primer used in real-time PCR analysis.

\begin{tabular}{|c|c|c|}
\hline Gene name & Forward primer & Reverse primer \\
\hline Sox 2 & GCCGAGTGGAAACTTTTGTCG & GGCAGCGTGTACTTATCCTTCT \\
\hline$c-k i t$ & CGTTCTGCTCCTACTGCTTCG & CCCACGCGGACTATTAAGTCT \\
\hline Oct3/4 & GGGAGATTGATAACTGGTGTGTT & GTGTATATCCCAGGGTGATCCTC \\
\hline Nanog & TTTGTGGGCCTGAAGAAAACT & AGGGCTGTCCTGAATAAGCAG \\
\hline$C D 133$ & AGTCGGAAACTGGCAGATAGC & GGTAGTGTTGTACTGGGCCAAT \\
\hline nestin & CTGCTACCCTTGAGACACCTG & GCTCTGATCTCTGCATCTAC \\
\hline Bmil & CGTGTATTGTTCGTTACCTGGA & CAGTAGTGGTCTGGTCTTGT \\
\hline ActinB & TGTTACCAACTGGGAAGACA & GGGGTGTTGAAGGTCTCAAA \\
\hline
\end{tabular}

A
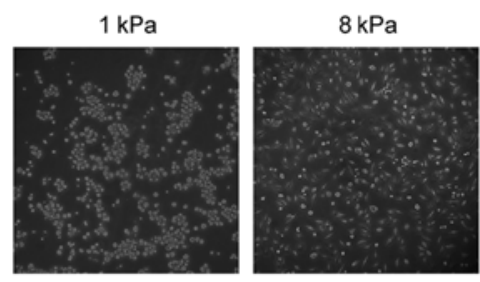

D

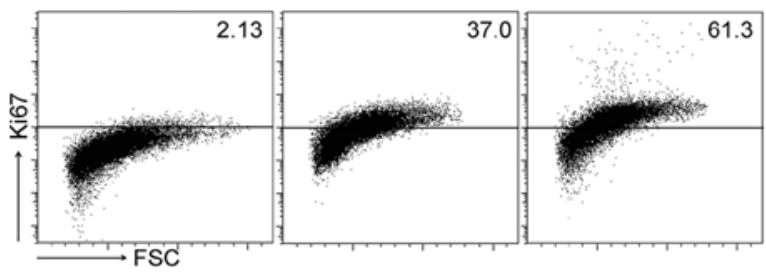

B

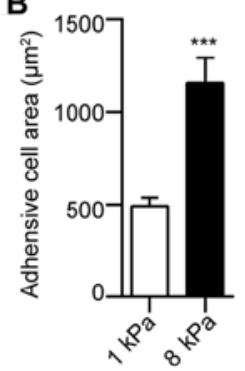

C

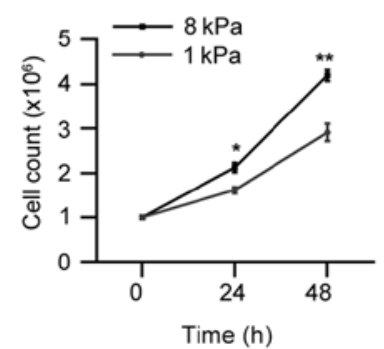

E

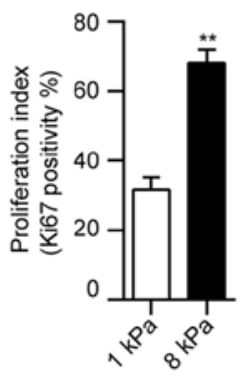

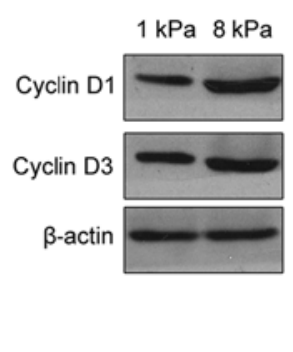

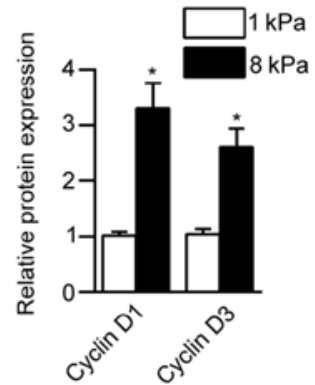

Figure 1. Matrix stiffness modulates Hep-2 laryngeal cancer cells growth and morphology. Hep-2 cells were cultured on collagen-I-coated polyacrylamide (PA) gels with 1 or $8 \mathrm{kPa}$ stiffness. (A) Morphology of Hep-2 on different stiffness substrates. (B) The surface area Hep-2 on different stiffness substrates was calculated by digital image analysis. (C) Hep-2 cell number was counted directly after 24- or 48-h culture on soft and stiff gels. (D) Flow cytometry analysis of Hep-2 cells stained with Ki67. (E) Whole-cell lysates were extracted from Hep-2 cells cultured on soft (1 kPa) and stiff (8 kPa) gels. The expression of cyclin D1 and cyclin D3 were determined by western blot analysis. $\beta$-actin serves as a loading control. Data are representative of three independent experiments; error bars, SEM, ${ }^{*} \mathrm{P}<0.05 ;{ }^{* *} \mathrm{P}<0.01 ;{ }^{* * *} \mathrm{P}<0.001$.

B1-integrinandphospho-FAKregulate the stiffness-dependent proliferation of Hep- 2 cells. Integrins and integrin-associated focal adhesions are known to be important mediators of mechanotransduction. Interestingly, we found that Hep-2 cells displayed an increase of the $\beta 1$-integrin (CD29) with increasing stiffness (Fig. 2A). Next, we analyzed the stiffness-dependent activity of critical signaling pathways using immunoblotting. We observed enhanced FAK, ERK, Akt and STAT3 phosphorylation in Hep-2 cell cultured on stiff $(8 \mathrm{kPa})$ versus soft (1 kPa) gels (Fig. 2B). To test whether $\beta 1$-integrin and phospho-FAK signal is responsible for the stiffnessdependent proliferation of Hep-2 cells, we first blocked the $\beta 1$-integrin signal using a functional anti-CD29 blocking antibody. Proliferation of Hep-2 cell cultured on $8 \mathrm{kPa}$ gels was significant reduced by treatment with anti-CD29 antibody relative to relevant controls (Fig. 2C). To investigate the effect of FAK activation on Hep-2 cell proliferation, we treated Hep- 2 cells cultured on $8 \mathrm{kPa}$ gel with the small molecular FAK inhibitor PF573228. FAK inhibitor markedly inhibited the proliferation of Hep-2 cells (Fig. 2D). Furthermore, we used siRNA to knock down $\beta 1$-integrin or FAK expression in Hep-2 cells and observed a significant reduction in cellular proliferation relative to control siRNA transfection (Fig. 2E and F).

Matrix stiffness regulates stem cell-associated genes in Hep-2 cells. Cancer cell cultured on soft stiffness is associated with some features of cancer stem cells. To determine whether matrix stiffness regulates stem-cell-associated genes in Hep-2 cells, we measured a panel of stem cell markers including Sox2, 
A
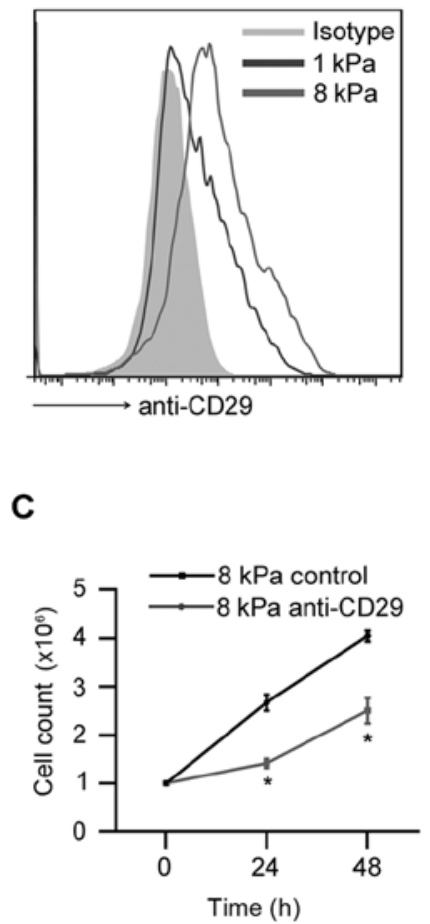

B

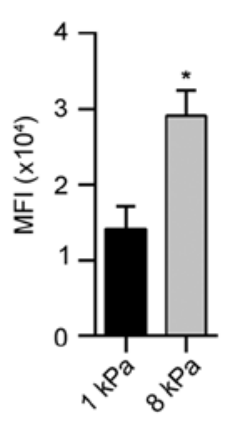

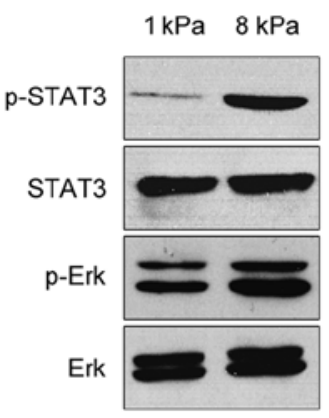
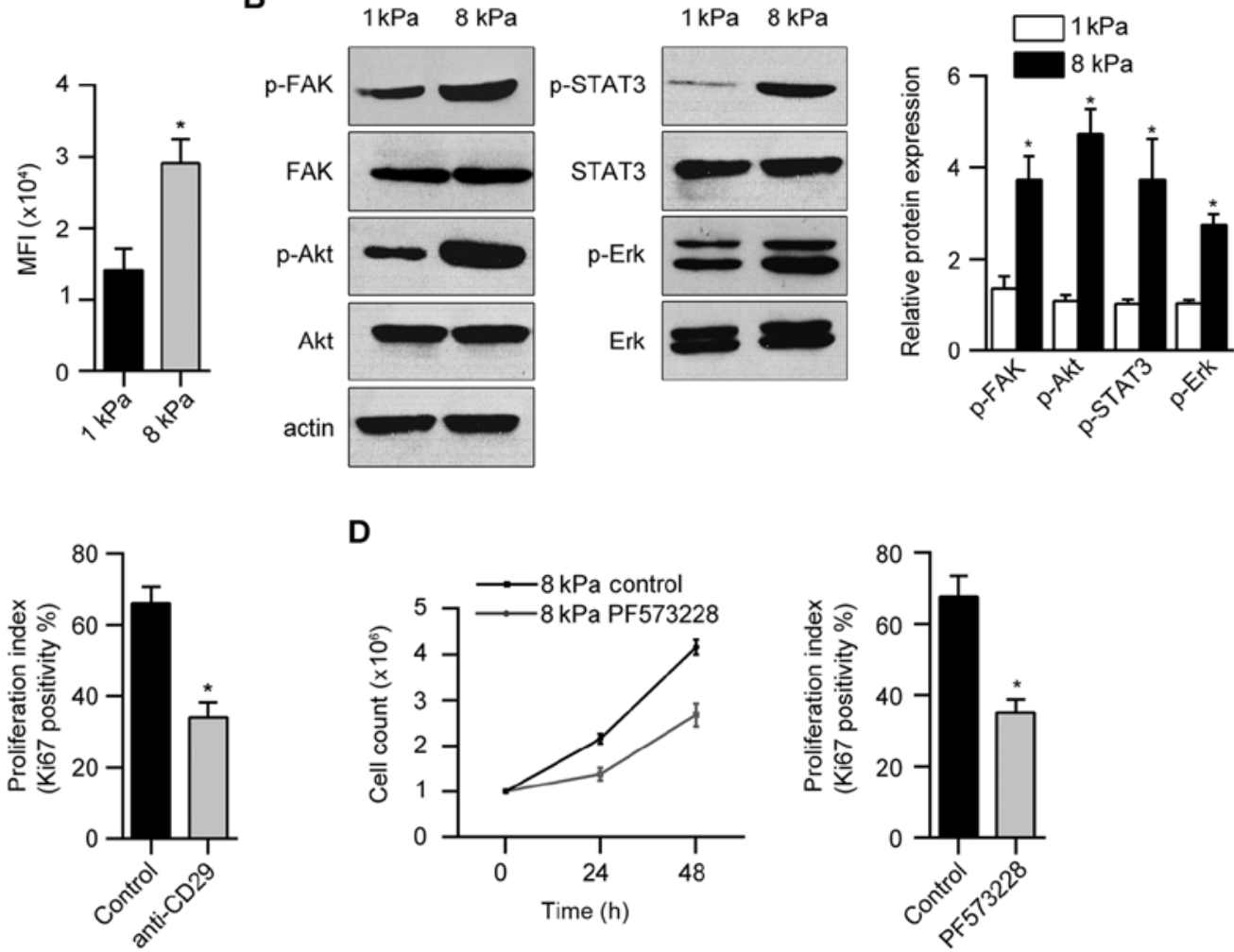

D
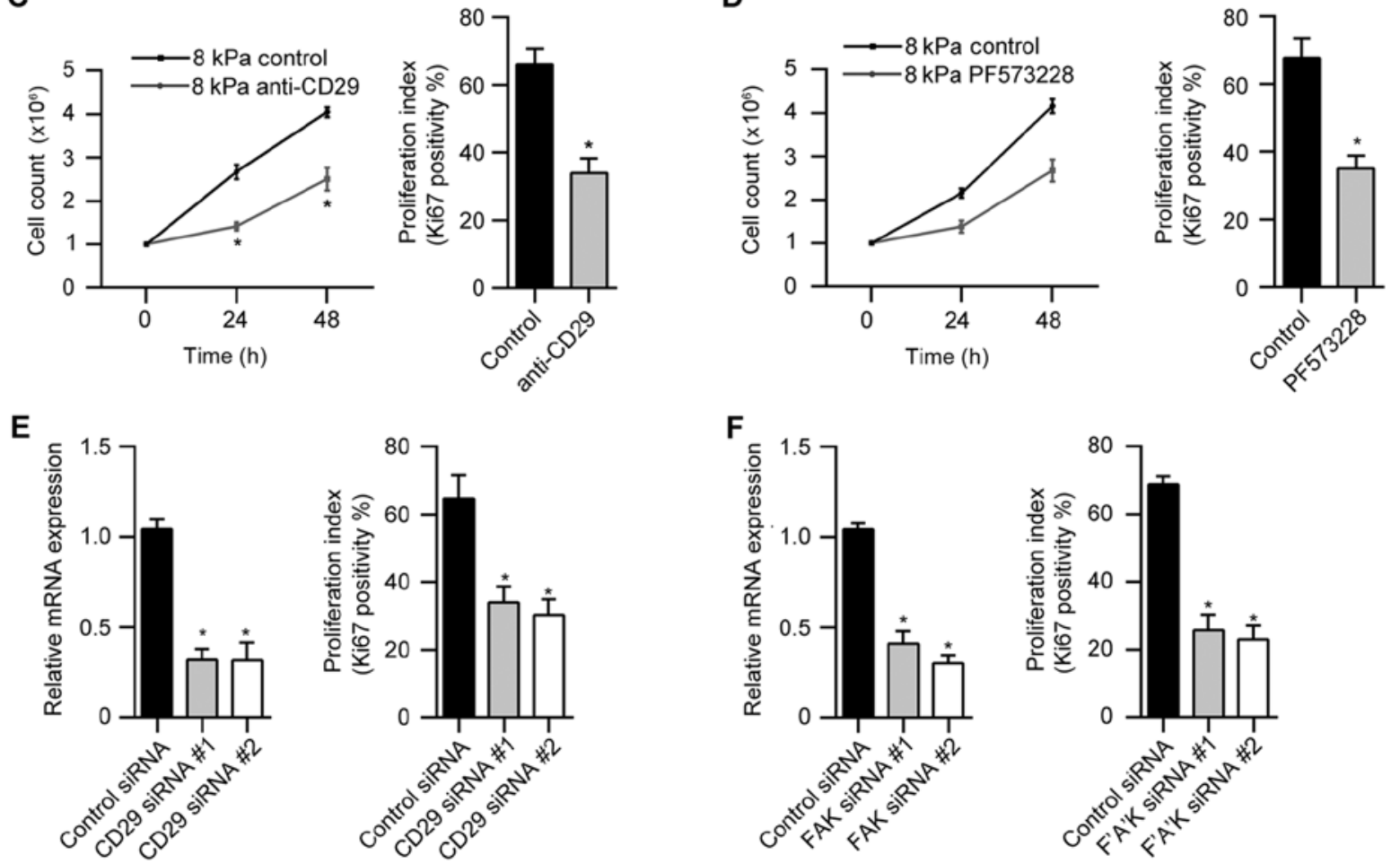

Figure 2. $\beta 1$-integrin and phospho-FAK regulate the stiffness-dependent proliferation of Hep-2 cells. (A) Flow cytometry analysis of Hep-2 cells cultured on soft $(1 \mathrm{kPa})$ and stiff $(8 \mathrm{kPa})$ gels stained with anti-CD29. Data are representative of three independent experiments. (B) Western blots showing expression of phosphorylated and total FAK, ERK, Akt and STAT3 in Hep-2 cells cultured on soft (1 kPa) and stiff ( $8 \mathrm{kPa})$ gels, as indicated. (C and D) Analyses of growth and proliferative index (Ki67 positivity) of Hep-2 cells cultured on $8 \mathrm{kPa}$ gel. Cells were treated with the anti-CD29 antibody (C). Cells were treated with the FAK inhibitor PF573228 for $24 \mathrm{~h}$ (D). (E and F) Hep-2 cells transfected with CD29 siRNA or FAK siRNA and then cultured on 8 kPa gel. Proliferative index (Ki67 positivity) was analyzed. Data are representative of three independent experiments; error bars, $\mathrm{SEM},{ }^{*} \mathrm{P}<0.05$.

Oct3/4, Nanog, CD133, nestin, Bmi-1 and c-kit using reverse transcriptase-PCR and real-time PCR (Fig. 3A and B). Hep-2 cell cultured on soft gel was associated with an increased stem cell-associated genes. We further performed flow cytometric analyses for putative cell-surface stem cell markers in Hep-2 cells cultured on soft and stiff gels. Fig. 3C demonstrated upregulation of cancer stem cell markers CD133 and c-kit in Hep-2 cells cultured on soft versus stiff gels. These results suggest that matrix stiffness regulates stem cell-associated genes in Hep-2 cells.

Matrix stiffness regulates chemosensitivity of Hep-2. Cancer stem cells are known as resistant to chemotherapeutic druginduced apoptosis. To investigate whether Hep2 cells cultured on soft gel are more drug-resistant, different concentrations of cisplatin were added to the culture medium. Following cisplatin treatment, an increased frequency of apoptotic cells was induced in Hep-2 cells from stiff gels, compared those from soft gels (Fig. 4A). To confirm the validity of this finding, we used a second chemotherapeutic agent, 5-fluorouracil (5-FU) to repeat the experiment. Consistent with the result with cisplatin, Hep-2 cells cultured on soft gels were more resistant to apoptosis induced by 5-FU (Fig. 4B). In addition, the cell cytotoxicity of Hep-2 cells was detected by CCK- 8 assay. Fig. $4 \mathrm{C}$ showed that Hep-2 cells in soft $(1 \mathrm{kPa})$ gel were more resistant to chemo-drugs than in stiff $(8 \mathrm{kPa})$ gel. To investigate the influence of matrix stiffness on the survival of Hep-2 tumor cells after chemotherapy, we performed a colony formation assay. Following cisplatin or 5-FU treatment on Hep- 2 cells on soft $(1 \mathrm{kPa})$ and stiff $(8 \mathrm{kPa})$ gels, the surviving 
A

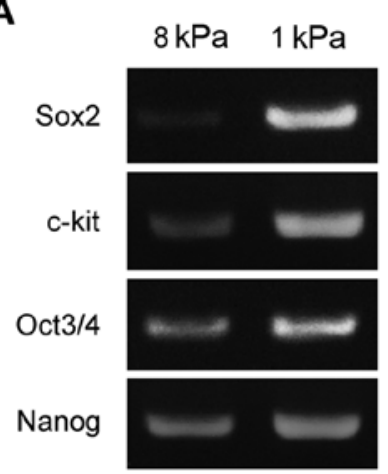

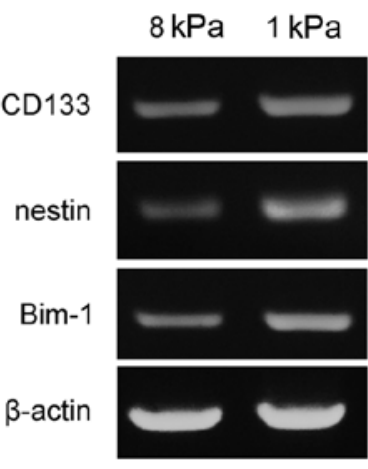

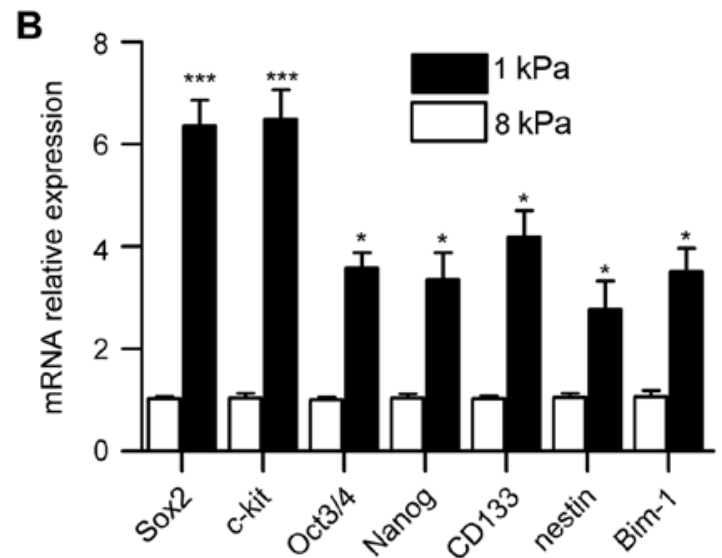

C
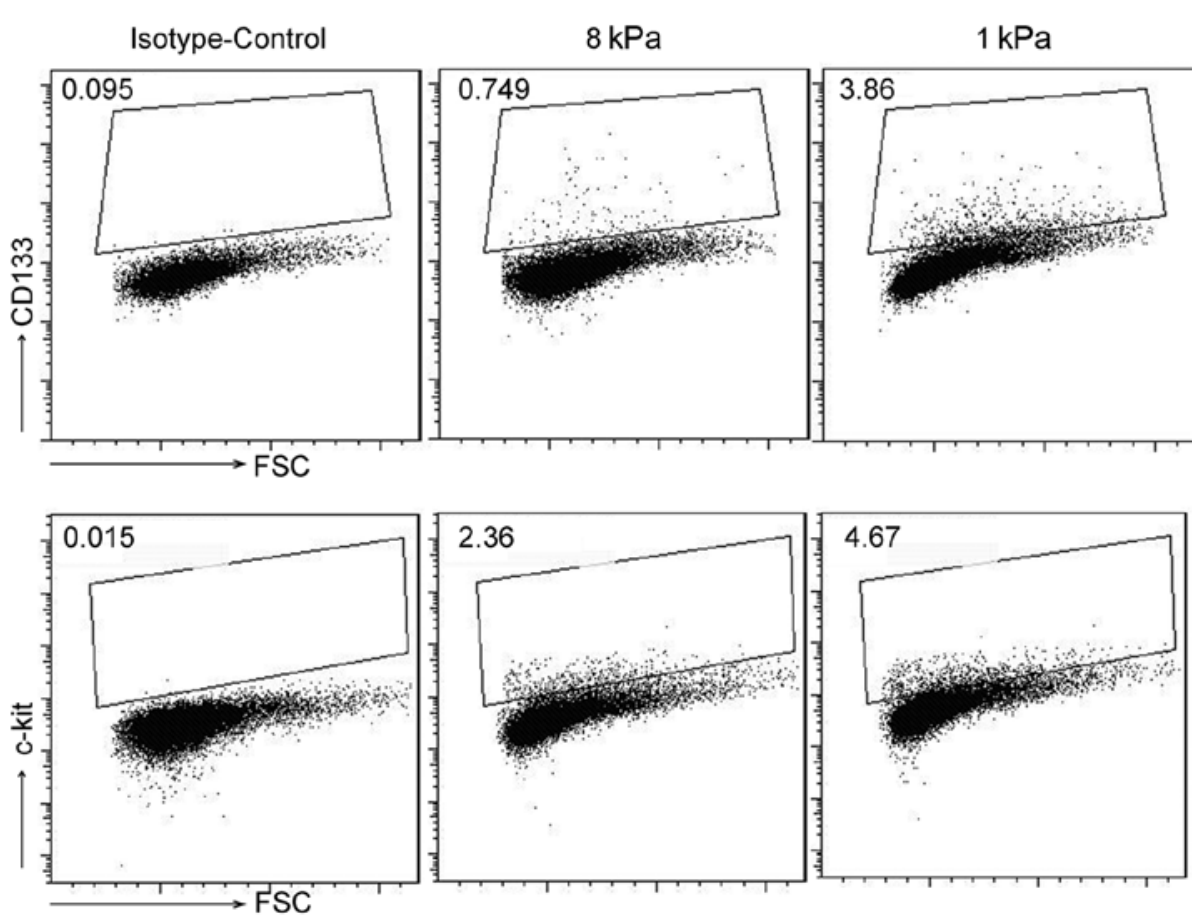

Figure 3. Matrix stiffness regulates stem cell-associated genes in Hep-2 cells. Hep-2 cells were cultured on soft (1 kPa) and stiff $(8 \mathrm{kPa}) \mathrm{gels}$ for $48 \mathrm{~h}$. Expression of stem cell-markers Sox2, Oct3/4, Nanog, CD133, nestin, Bmi-1 and c-kit were analyzed by reverse transcriptase-PCR (A) and real-time PCR (B). (C) CD133 and c-kit expression were analyzed by flow cytometry. Data are representative of three independent experiments; error bars, SEM, ${ }^{*} \mathrm{P}<0.05 ;{ }^{* * *} \mathrm{P}<0.001$.

Hep-2 cell population from soft gel formatted an increased colony cultured on rigid 12-well plates (Fig. 4D). These results suggest that matrix stiffness regulates chemosensitivity of Hep-2 cells, which is consistent with the expression of stem cell-associated markers.

Sox2 is essential for the chemoresistance of Hep-2 cells cultured in soft gels. Sox 2 plays an essential role in the maintenance of self-renewal of cancer stem cells. In addition, overexpression of Sox 2 promotes the migration and invasion in laryngeal squamous cancer cells. To verify the possible correlation between Sox 2 expression and chemoresistance of Hep-2 cells, we knocked down Sox2 in Hep-2 via siRNA interference and then cultured in $1 \mathrm{kPa}$ gels. Scrambled control siRNA treated cells were used as control. The knockdown of Sox 2 expression in Hep-2 cells was confirmed by real-time PCR and western blot analysis (Fig. 5A). Thirty-six hours after culture in $1 \mathrm{kPa}$ gels, Hep- 2 cells were treated with $10 \mu \mathrm{M}$ cisplatin. Flow cytometry and CCK8 assay showed that silencing of Sox 2 in Hep- 2 cells on soft gel significantly decreased the chemoresistance ability when compared with scrambled control (Fig. 5B and C). Similarly, Sox2 siRNA decreased the chemoresistance ability of Hep-2 cells when treated with $10 \mu \mathrm{M}$ 5-fluorouracil (Fig. 5B and C).

Sox 2 promotes chemoresistance of Hep-2 cells in soft stiffness via upregulating the expression of $A B C G 2$. ATP-binding cassette (ABC) transporters are well-known to exclude a wide variety of chemotherapeutic drugs from the cytoplasm, thereby leading to multidrug resistance. Among various $\mathrm{ABC}$ transporters, ABCG2 is thought to play an important role in laryngeal squamous cancer cells. Interestingly, Hep-2 cells cultured in soft gel expressed significant high level of ABCG2 compared with Hep-2 in stiff gel (Fig. 6A and B). In addition, 
A
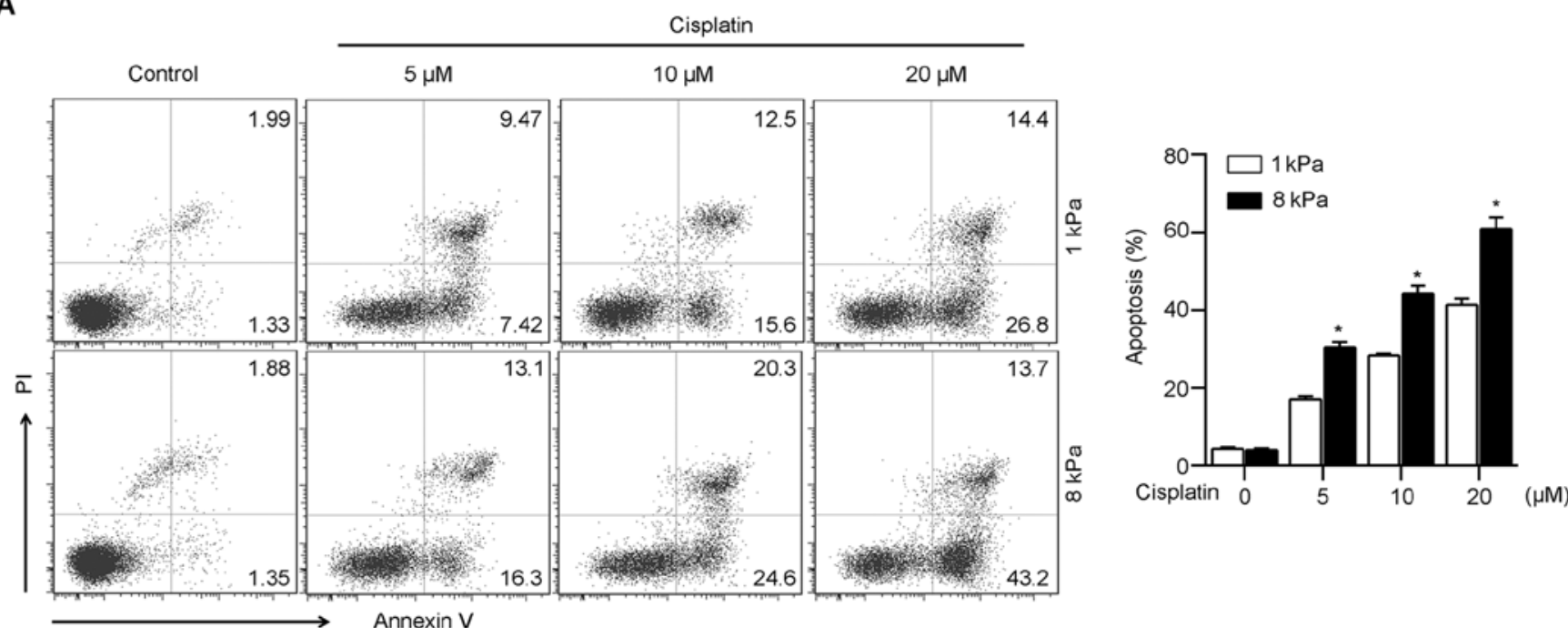

B
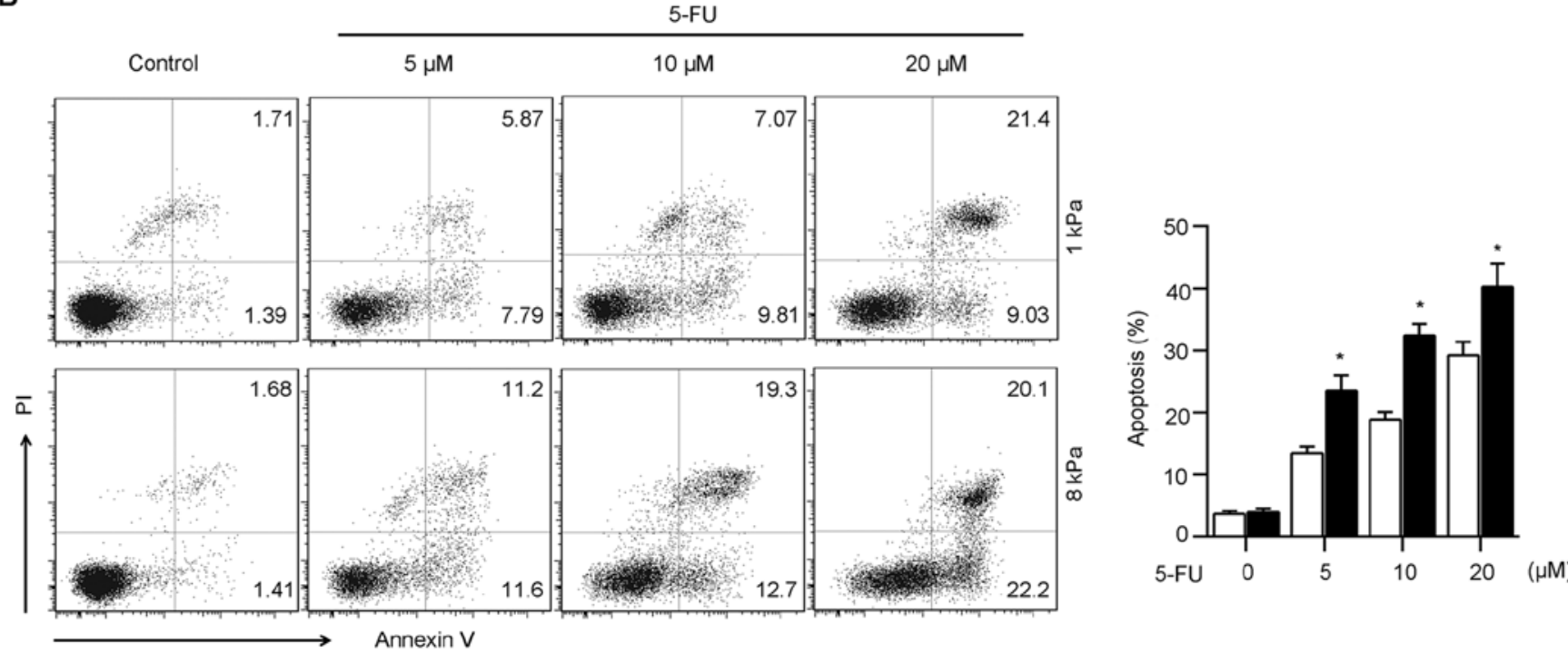

C
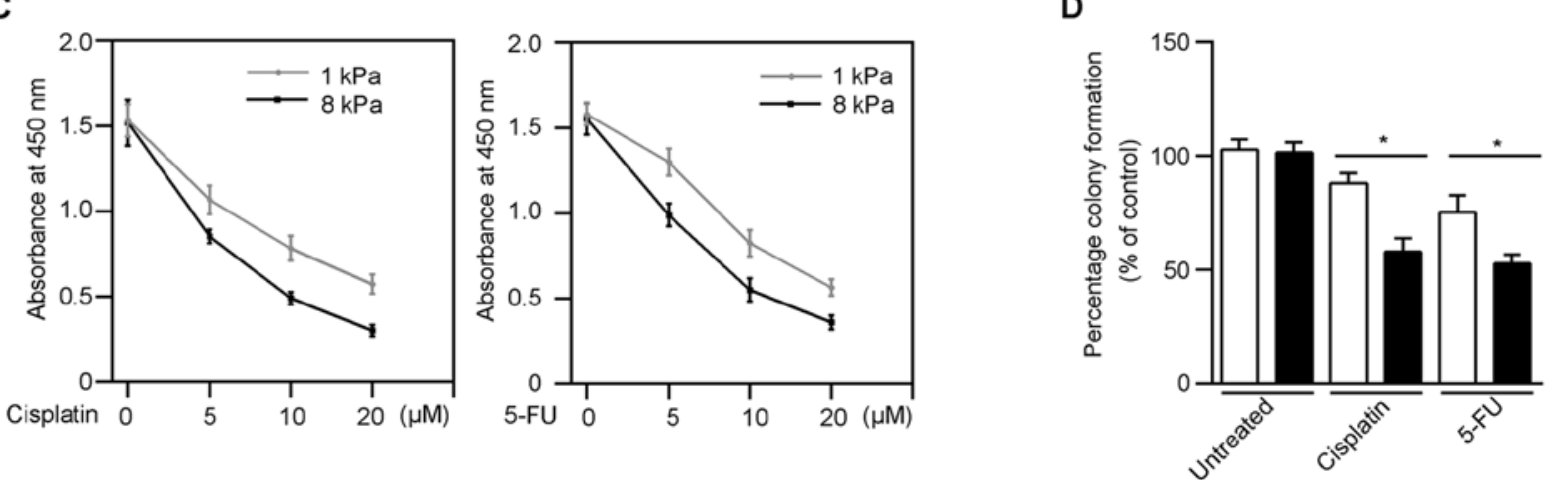

Figure 4. Matrix stiffness regulates chemosensitivity of Hep-2. (A) Flow cytometry analysis of cisplatin treated Hep-2 cells stained with Annexin V and PI. (B) Flow cytometry analysis of 5-FU treated Hep-2 cells stained with Annexin V and PI. (C) Hep-2 cells cultured on soft (1 kPa) and stiff ( $8 \mathrm{kPa})$ gels were treated with various concentrations of cisplatin or 5-FU. Cell cytotoxicity was detected by CCK-8 assay. (D) Percentage of colony formation Hep-2 cells after cisplatin or 5-FU treatment. Hep-2 cells were cultured for $48 \mathrm{~h}$ on either 1 or $8 \mathrm{kPa}$ gels. Cells were then left untreated or treated with cisplatin or 5 -fluorouracil for $24 \mathrm{~h}$. Then the medium was changed and cultured for a further $48 \mathrm{~h}$. Cells were trypsinized and equal numbers re-plated at clonal density in 12 -well plates. Percentage of colony formation was calculated by direct counting of the resulting colonies. Data are representative of three independent experiments; error bars, SEM, ${ }^{*} \mathrm{P}<0.05$.

silencing of Sox 2 in Hep-2 cells resulted in downregulation of ABCG2 compared with control cells (Fig. 6C). Furthermore, we knocked down ABCG2 in Hep-2 using siRNA and then cultured in $1 \mathrm{kPa}$ gels (Fig. 6D). Downregulation of ABCG2 
A

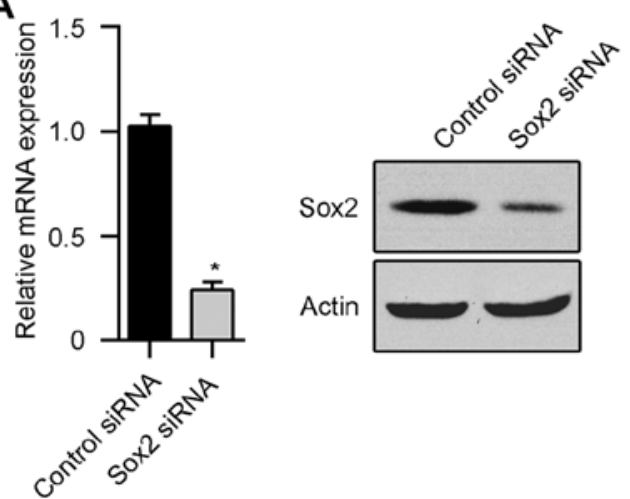

B

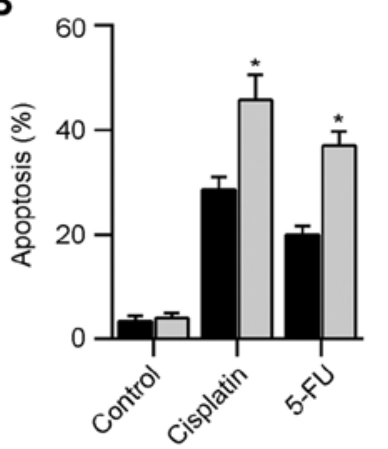

C

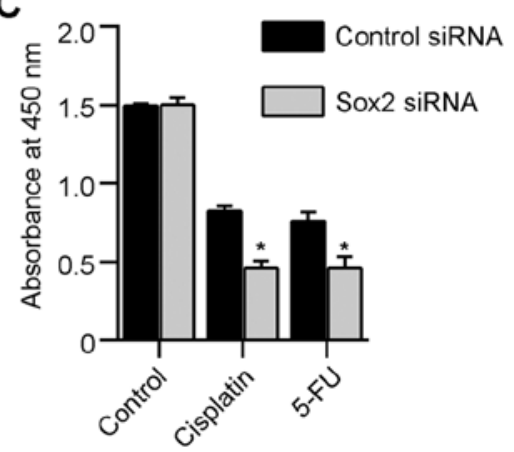

Figure 5. Sox 2 is essential for the chemoresistance of Hep-2 cells cultured in soft stiffness. (A) siRNAs were transfected into Hep-2 using Lipofectamine RNAiMAX. Forty-eight hours after transfection, mRNA of Sox 2 were detected by real-time PCR (left panel). Seventy-two hours after transfection, protein level of Sox 2 were detected by western blot analysis (right panel). (B) Hep-2 cells transfected with Sox 2 siRNA and cultured on 1 kPa gels. Flow cytometry analysis of cisplatin or 5-FU treated Hep-2 cells stained with Annexin V and PI. (C) Hep-2 cells were treated as (B) and cell cytotoxicity was detected by CCK-8 assay. Data are representative of three independent experiments; error bars, $\mathrm{SEM},{ }^{*} \mathrm{P}<0.05$

A

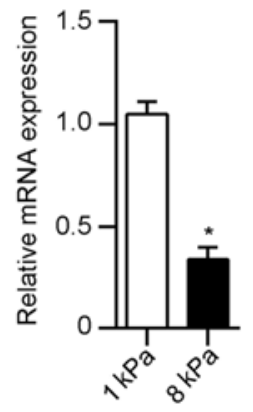

B

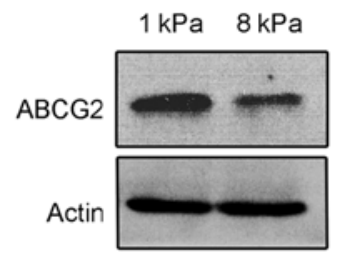

C

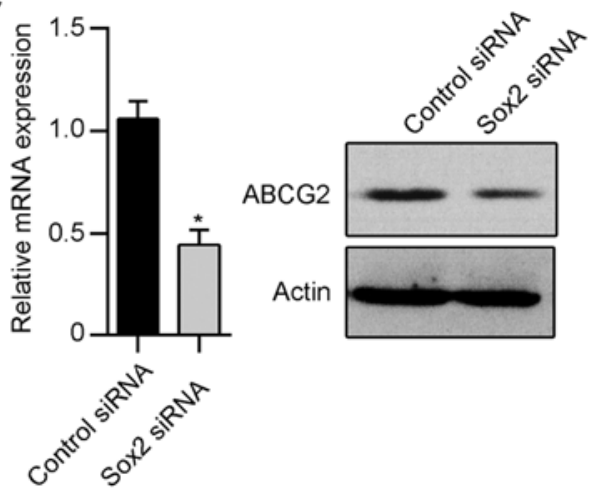

E

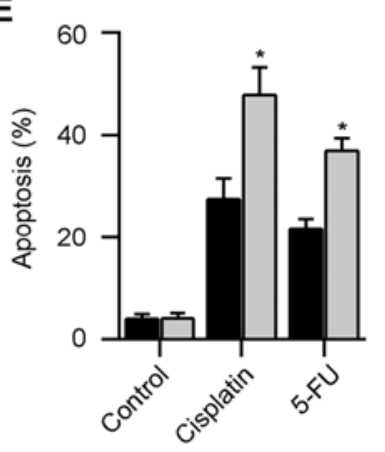

F

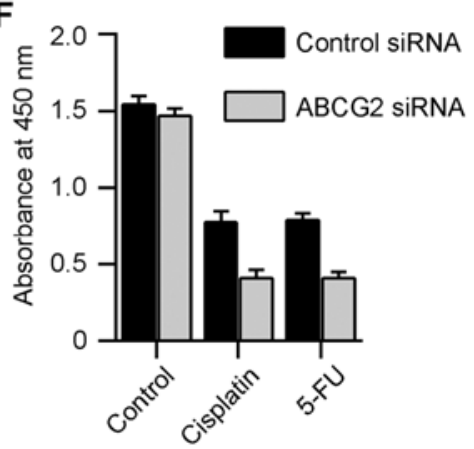

Figure 6. Sox 2 promotes chemoresistance of Hep-2 cells in soft stiffness via upregulating the expression of ABCG2. (A) ABCG2 expression of Hep-2 cells cultured on soft $(1 \mathrm{kPa})$ and stiff $(8 \mathrm{kPa})$ gels were detected by real-time PCR. (B) Western blots showing expression of ABCG2 in Hep-2 cells cultured on soft $(1 \mathrm{kPa})$ and stiff $(8 \mathrm{kPa})$ gels. (C) Hep-2 cells transfected with Sox 2 siRNA and cultured on $1 \mathrm{kPa}$ gels. Seventy-two hours later, mRNA level and protein level of Sox 2 were detected. (D) siRNAs were transfected into Hep-2 cells using Lipofectamine RNAiMAX. Forty-eight hours after transfection, mRNA of ABCG2 were detected by real-time PCR (left panel). Seventy-two hours after transfection, protein level of ABCG2 were detected by western blot analysis (right panel). (E) Hep-2 cells transfected with ABCG2 siRNA and cultured on $1 \mathrm{kPa}$ gels. Flow cytometry analysis of cisplatin or 5-FU treated Hep-2 cells stained with Annexin V and PI. (F) Hep-2 cells were treated as (E) and cell cytotoxicity was detected by CCK- 8 assay. Data are representative of three independent experiments; error bars, SEM, ${ }^{*} \mathrm{P}<0.05$.

in Hep-2 cells cultured in soft gels restored drug sensitivity after cisplatin and 5-FU treatment (Fig. 6E and F). These data suggest that Sox 2 has a major role in drug resistance of Hep- 2 cells in soft gels by regulating the ABCG2 expression.

\section{Discussion}

Mechanical forces play an essential role in the signal transduction at cell-matrix and cell-cell contacts, modulating various 
cellular behaviors such as cell adhesion, proliferation, migration, and differentiation (16-18). In this study, we demonstrated that the stiffness of the external matrix markedly alters the phenotype and behavior of laryngeal squamous cell carcinoma cells in vitro. It has previously been demonstrated that matrix stiffness can regulate proliferation in glioma cells (19) and hepatocellular carcinoma cells (9). Our data supported that stiff substrate significantly promote the proliferation of Hep-2 cells, verified by direct counting and Ki67 staining. In accordance with these findings, we found that the expression of cyclin D1 and cyclin D3 were upregulated in Hep-2 cells on $8 \mathrm{kPa}$ gel. Focal adhesion, an integrin-based adhesion complex, regulates cellular behavior in various biological context by transferring external force information in a bidirectional manner (20). Integrin-FAK signaling pathway is responsible for mechanotransduction (21). Interestingly, we found that Hep-2 cells on stiff gels express higher level of $\beta 1$-integrin. Consistently, we have demonstrated that $\beta 1$-integrin and FAK activation regulate stiffness-dependent proliferation in Hep-2 cells. Treatment with anti-CD29 or FAK inhibitor PF573228, markedly decreased the proliferation of Hep-2 cells on stiff gel. Additionally, we observed an increase in the magnitude of ERK, Akt, and STAT3 activation in Hep-2 cells cultured on stiff gels, suggesting that increasing matrix stiffness promoted activity of critical mitogenic signaling pathways.

Previous studies showed that soft matrix upregulate stem-cell-associated genes in B16 or HCC cells. Most strikingly, Liu et al showed that a few B16 cells cultured in soft fibrin gel led to the formation of solid tumors in syngeneic or severe combined immunodeficiency mice (14). In the present study, Hep-2 cells on soft gel expressed higher level of stem cell markers (Sox2, Oct3/4, Nanog, CD133, nestin, Bmi-1 and c-kit). However, the underlying mechanisms regulating the expression of stem cell genes by mechanical force needs to be further determined. A possible mechanism elucidated by Tajik et al (15) is that externally mechanical force induced the direct stretching of chromatin, leading to the transcription upregulation.

It is known that cancer stem cells are more resistant to chemotherapeutic drug-mediated cell death than differentiated cancer cells $(22,23)$. Intriguingly, we demonstrated that Hep-2 cells on soft gel are more resistant to chemotherapeutic drug than those from stiff gel, which is consistent with previous study on HCC cells. A recent study showed that Sox 2 played a regulatory role in the growth, migration and invasion of LSCC (24). We showed that Sox 2 is essential for the chemoresistance of Hep-2 cells cultured in soft gels. Knockdown of Sox 2 using siRNA significant increased the drug-sensitivity of Hep-2 cells on soft gels. ATP-binding cassette (ABC) transporter is known to exclude drugs from cytoplasm to extracellular environment. Here, we provide further evidence that Sox 2 promote chemoresistance through upregulating the expression of $\mathrm{ABCG} 2$, an $\mathrm{ABC}$ transporter which plays an essential role in LSCC.

In conclusion, our study shows that increasing matrix stiffness promotes the proliferation of Hep-2 cells, in turn, Hep-2 cells cultured on soft substrate expressed higher level of stem cell markers. Especially, the Sox 2 upregulation in Hep-2 cells on soft gel induced the expression ABCG2, resulting in resistance to chemotherapeutic drugs. Our present study provides a new mechanism of the growth and behavior of LSCC. Thus, these findings will provide a new platform for the future design of anticancer drugs based on the biophysical properties of the tumor site.

\section{Acknowledgements'}

This study was supported by Natural Science Foundation of Liaoning Province of China (no. 201202287).

\section{References}

1. Marioni G, Marchese-Ragona R, Cartei G, Marchese F and Staffieri A: Current opinion in diagnosis and treatment of laryngeal carcinoma. Cancer Treat Rev 32: 504-515, 2006.

2. Papadas TA, Alexopoulos EC, Mallis A, Jelastopulu E, Mastronikolis NS and Goumas P: Survival after laryngectomy: A review of 133 patients with laryngeal carcinoma. Eur Arch Otorhinolaryngol 267: 1095-1101, 2010.

3. Chai LP, Wang ZF, Liang WY, Chen L, Chen D, Wang AX and Zhang ZQ: In vitro and in vivo effect of 5-FC combined gene therapy with TNF- $\alpha$ and CD suicide gene on human laryngeal carcinoma cell line Hep-2. PLoS One 8: e61136, 2013

4. Paszek MJ, Zahir N, Johnson KR, Lakins JN, Rozenberg GI, Gefen A, Reinhart-King CA, Margulies SS, Dembo M, Boettiger $\mathrm{D}$, et al: Tensional homeostasis and the malignant phenotype. Cancer Cell 8: 241-254, 2005.

5. Levental KR, Yu H, Kass L, Lakins JN, Egeblad M, Erler JT, Fong SF, Csiszar K, Giaccia A, Weninger W, et al: Matrix crosslinking forces tumor progression by enhancing integrin signaling. Cell 139: 891-906, 2009.

6. Samuel MS, Lopez JI, McGhee EJ, Croft DR, Strachan D, Timpson P, Munro J, Schröder E, Zhou J, Brunton VG, et al: Actomyosin-mediated cellular tension drives increased tissue stiffness and $\beta$-catenin activation to induce epidermal hyperplasia and tumor growth. Cancer Cell 19: 776-791, 2011.

7. Frantz C, Stewart KM and Weaver VM: The extracellular matrix at a glance. J Cell Sci 123: 4195-4200, 2010.

8. Lu P, Weaver VM and Werb Z: The extracellular matrix: A dynamic niche in cancer progression. J Cell Biol 196: 395-406, 2012.

9. Schrader J, Gordon-Walker TT, Aucott RL, van Deemter M, Quaas A, Walsh S, Benten D, Forbes SJ, Wells RG and Iredale JP: Matrix stiffness modulates proliferation, chemotherapeutic response, and dormancy in hepatocellular carcinoma cells. Hepatology 53: 1192-1205, 2011.

10. Yang $\mathbf{J}$ and Weinberg RA: Epithelial-mesenchymal transition: At the crossroads of development and tumor metastasis. Dev Cell 14: 818-829, 2008.

11. Thiery JP, Acloque H, Huang RY and Nieto MA: Epithelialmesenchymal transitions in development and disease. Cell 139: 871-890, 2009.

12. Wei SC, Fattet L, Tsai JH, Guo Y, Pai VH, Majeski HE, Chen AC, Sah RL, Taylor SS, Engler AJ, et al: Matrix stiffness drives epithelial-mesenchymal transition and tumour metastasis through a TWIST1-G3BP2 mechanotransduction pathway. Nat Cell Biol 17: 678-688, 2015.

13. Chowdhury F, Li Y, Poh YC, Yokohama-Tamaki T, Wang N and Tanaka TS: Soft substrates promote homogeneous self-renewal of embryonic stem cells via downregulating cell-matrix tractions. PLoS One 5: e15655, 2010.

14. Liu J, Tan Y, Zhang H, Zhang Y, Xu P, Chen J, Poh YC, Tang K, Wang $\mathrm{N}$ and Huang B: Soft fibrin gels promote selection and growth of tumorigenic cells. Nat Mater 11: 734-741, 2012.

15. Tajik A, Zhang Y, Wei F, Sun J, Jia Q, Zhou W, Singh R, Khanna N, Belmont AS and Wang N: Transcription upregulation via force-induced direct stretching of chromatin. Nat Mater 15: 1287-1296, 2016

16. Jaalouk DE and Lammerding J: Mechanotransduction gone awry. Nat Rev Mol Cell Biol 10: 63-73, 2009.

17. Calvo F, Ege N, Grande-Garcia A, Hooper S, Jenkins RP, Chaudhry SI, Harrington K, Williamson P, Moeendarbary E, Charras G, et al: Mechanotransduction and YAP-dependent matrix remodelling is required for the generation and maintenance of cancer-associated fibroblasts. Nat Cell Biol 15: 637-646, 2013. 
18. Butcher DT, Alliston T and Weaver VM: A tense situation: Forcing tumour progression. Nat Rev Cancer 9: 108-122, 2009.

19. Yang YL, Motte S and Kaufman LJ: Pore size variable type I collagen gels and their interaction with glioma cells. Biomaterials 31: 5678-5688, 2010.

20. Kanchanawong P, Shtengel G, Pasapera AM, Ramko EB Davidson MW, Hess HF and Waterman CM: Nanoscale architecture of integrin-based cell adhesions. Nature 468: 580-584, 2010.

21. Palazzo AF, Eng CH, Schlaepfer DD, Marcantonio EE and Gundersen GG: Localized stabilization of microtubules by integrin- and FAK-facilitated Rho signaling. Science 303: 836-839, 2004
22. Dean M, Fojo T and Bates S: Tumour stem cells and drug resistance. Nat Rev Cancer 5: 275-284, 2005.

23. Cojoc M, Mäbert K, Muders MH and Dubrovska A: A role for cancer stem cells in therapy resistance: Cellular and molecular mechanisms. Semin Cancer Biol 31: 16-27, 2015.

24. Yang N, Hui L, Wang Y, Yang H and Jiang X: SOX2 promotes the migration and invasion of laryngeal cancer cells by induction of MMP-2 via the PI3K/Akt/mTOR pathway. Oncol Rep 31: 2651-2659, 2014. 